PFC/JA-92-16

\author{
Bootstrap Current Induced by Fusion Born \\ Alpha Particles \\ C. T. Hsu, K. T. Shaing, ${ }^{*}$ D. J. Sigmar, R. Gormley
}

\author{
Plasma Fusion Center \\ Massachusetts Institute of Technology \\ Cambridge, MA 02139 USA
}

This work was supported by the US Department of Energy under contract DE-FG02-91ER-

54109. Reproduction, translation, publication, use, and disposal, in whole or in part, by or for the US Government is permitted.

Submitted for publication in: Physics of Fluids B

* Oak Ridge National Laboratory, Oak Ridge, TN 37831 


\title{
BOOTSTRAP CURRENT INDUCED BY FUSION BORN
}

\section{ALPHA PARTICLES}

C. T. HSU, R. P. GORMLEY, D. J. SIGMAR

Massachusetts Institute of Technology, Plasma Fusion Center, 167 Albany Street, NW16-260, Cambridge, MA 02139 USA, Tel: (617) 253-2470

K. C. SHAING

Oak Ridge National Laboratory, Oak Ridge, TN 37831

\begin{abstract}
The bootstrap current produced by fusion born alpha particles is obtained retaining effects of slowing down drag, pitch angle scattering, and arbitrary aspect ratio. The result is presented both as a summation of a rapidly converging series and a simple Padé approximation good for arbitrary aspect ratio. Quantitative results are derived using International Thermonuclear Experimental Reactor (ITER) ${ }^{1}$ parameters.
\end{abstract}




\section{INTRODUCTION}

In toroidal plasma systems, the parallel friction force will attempt to equilibrate the parallel flows of different species by generating a relative poloidal flow in response to the relative parallel diamagnetic flow. A finite parallel relative flow remains due to existence of magnetically trapped orbits since they cannot contribute to the poloidal flows. This effect leads to a current, usually referred to as the bootstrap current which is proportional to the relative parallel diamagnetic return flow times the fraction of trapped particles.

The neoclassical bootstrap current is of great importance for future tokamak fusion devices because at sufficient poloidal beta it can provide a substantial fraction of the total current. Typically, the fusion produced fast $\alpha$ particles can produce a beta gradient comparable to that of the background plasma. This implies that fast $\alpha$ particles may result in a bootstrap current contribution comparable with the background bootstrap current.

Previously, the $\alpha$ particle induced bootstrap current had been calculated ${ }^{2,3}$ by keeping only the drag term in the collision operator and was found to be small because it arose only due to "banana collapsing" during the slowing down process, which scales as $\propto \epsilon^{3 / 2}$ ( $\epsilon$ is the inverse aspect ratio). However, in recent work, ${ }^{4}$ it has been found that pitch angle scattering can be very important in the neoclassical transport process of fast $\alpha$ particles and leads to transport fluxes $\propto \epsilon^{1 / 2}$. In this work, the bootstrap current will be calculated based on the work of Ref. 4 in which both drag and pitch angle scattering collisional effects are included for the $\alpha$ particle kinetics.

By imposing the quasineutrality condition, the plasma current can be written as

$$
\mathbf{J}=e\left[n_{e} \mathbf{V}_{i e}+n_{\alpha} Z_{\alpha} \mathbf{V}_{\alpha i}+\sum_{I} n_{I} Z_{I} \mathbf{V}_{I i}\right]
$$

where the subscript $i$ refers to the main ion, and $I$ refers to impurities and $\mathbf{V}_{i j} \equiv \mathbf{V}_{\boldsymbol{i}}-\mathbf{V}_{j}$ is the difference of flows between species $i$ and $j$. Hence, the current can be obtained upon determining the relative flow velocities $\mathbf{V}_{i e}, \mathbf{V}_{\alpha i}$, and $\mathbf{V}_{I i}$.

It will be shown that the dynamics of all bulk plasma ion species (i.e., $i, I$ ) are hardly influenced by $\alpha$ particles, and thus can be treated as known quantities which can be found 
in the existing literature. Thus in the next two sections, only the $\alpha$ particle parallel flow and electron parallel flow will be calculated. In Section IV, the bootstrap current will be calculated and the quantitative results will be given based on ITER parameters. Conclusions are given in Section V.

\section{CALCULATION OF $V_{\alpha i}$}

In Ref. 4 , the $\alpha$ particle response was calculated by including the effects of finite aspect ratio and both the drag and pitch angle scattering. Nonetheless, ion flow and $\mathbf{E} \times \mathbf{B}$ drift was neglected. Although this can be justified due to the fact that

$$
\frac{V_{i}}{V_{\alpha}} \sim \frac{T_{i}}{E_{\alpha}} \ll 1,
$$

it is still interesting to include them (i) for completeness and (ii) to demonstrate that the $\alpha$ transport flux is independent of $E_{r}$.

By considering also the fact that the fusion source term is isotropic in the ion rest frame instead of the lab frame, one finds that it is appropriate to treat the $\alpha$ particle kinetic equation in the ion rest frame. In Appendix A, the $\alpha$ drift kinetic equation in the ion rest frame is obtained. The resulting drift kinetic equation (A8) is then solved by following the same procedure as in Ref. 4 (also cf. Appendix A) which yields the first order distribution function

$$
f_{\alpha 1}=-\frac{I v_{\|}}{\Omega_{\alpha}} \frac{\partial}{\partial \psi} f_{\alpha 0}+v_{\|} V_{\| i}^{*} \frac{\partial}{\partial w} f_{\alpha 0}+P(\lambda, w, \psi)
$$

with

$$
P(\lambda, w, \psi)=\sum_{j=1,3}\left(\sum_{n=1}^{\infty} \Lambda_{n}(\lambda, \psi) V_{n j}(w, \psi)\right) A_{j}(w, \psi) .
$$

Here, $v$ is the $\alpha$ particle velocity in the ion rest frame, $w \equiv \frac{v^{2}}{2} ; \lambda \equiv \frac{v_{1}^{2}}{v^{2}} h ; h \equiv \frac{B_{0}}{B} ; \Omega_{j} \equiv$ $\frac{Z_{j e B} B}{m_{j} c} ; I \equiv \mathrm{B} \cdot R^{2} \nabla \phi ; V_{\| i}^{*} \equiv \frac{-I}{m_{i} \Omega_{i} n_{i}} \frac{\partial}{\partial \psi} p_{i}+K_{i} B$ is the ion parallel flow less the $\mathbf{E} \times \mathbf{B}$ drift induced return flow; $K_{i} \equiv \frac{v_{i} \cdot \nabla \theta}{B \cdot \nabla \theta}$ corresponds to the ion poloidal flow; $p_{i}$ is the ion pressure; $\Lambda_{n}$ is the eigenfunction of the pitch angle scattering operator as given in Ref. 4; and

$$
f_{\alpha 0}(v, \psi)=\frac{S \tau_{s}}{4 \pi\left(v^{3}+v_{c}^{3}\right)} H\left(v_{0}-v\right)
$$


is the lowest order (slowing down) distribution function driven by the fusion source $S$. $P(\lambda, w, \psi)$ represents the localized distribution function which vanishes in the trapped region.

Denoting the flux surface average by \langle\rangle , the three driving forces for $P(\lambda, w, \psi)$ are

$$
\begin{aligned}
& A_{1} \equiv-\frac{I v}{2 \Omega_{0}} \frac{\partial}{\partial \psi} f_{\alpha 0} \\
& A_{2} \equiv\left\langle\frac{V_{\| i}^{*}}{h}\right\rangle \frac{1}{2} \frac{\partial}{\partial v} f_{\alpha 0} \\
& A_{3} \equiv-\frac{Z_{\alpha} e}{m_{\alpha}}\left\langle\frac{E_{\|}^{*}}{h}\right\rangle \frac{\tau_{s}}{2} \frac{\partial}{\partial v} f_{\alpha 0},
\end{aligned}
$$

and the corresponding $V_{n j}$ are (cf. Appendix A)

$$
\begin{aligned}
& V_{n 1}=\sigma_{n}\left[1-\frac{\left(\kappa_{n}-1\right) v_{b}^{3}}{\left(v_{c}^{3}+v^{3}\right) v \frac{\partial f_{\alpha 0}}{\partial \psi}} \int_{v}^{v_{0}} d u\left(\frac{v^{3}\left(v_{c}^{3}+u^{3}\right)}{u^{3}\left(v_{c}^{3}+v^{3}\right)}\right)^{\frac{Q_{p} \kappa_{n}}{3}} \frac{\partial f_{\alpha 0}(u)}{\partial \psi}\right] \\
& V_{n 2}=\sigma_{n}\left[1-\frac{\left(\kappa_{n}-1\right) v_{b}^{3}}{\left(v_{c}^{3}+v^{3}\right) \frac{\partial f_{\alpha 0}}{\partial u}} \int_{v}^{v_{0}} d u\left(\frac{v^{3}\left(v_{c}^{3}+u^{3}\right)}{u^{3}\left(v_{c}^{3}+v^{3}\right)}\right)^{\frac{Q_{p} \kappa_{n}}{3}} \frac{\partial f_{\alpha 0}(u)}{\partial u}\right] \\
& V_{n 3}=\frac{-\sigma_{n}}{\left(v^{3}+v_{c}^{3}\right) \frac{\partial f_{\alpha 0}}{\partial v}} \int_{v}^{v_{0}} d u\left(\frac{v^{3}\left(u_{3}+v_{c}^{3}\right)}{u^{3}\left(v^{3}+v_{c}^{3}\right)}\right)^{\frac{Q_{p} \kappa_{n}}{3}} u^{2} \frac{\partial f_{\alpha 0}(u)}{\partial u} .
\end{aligned}
$$

Here, $Q_{p}, \kappa_{n}$ and $\sigma_{n}$ are given in Ref. 4; in particular, $Q_{p} \equiv v_{b}^{3} / v_{b}^{3} \sim O(1)$ for D-T fusion.

$$
E_{\|}^{*}=E_{\|}-\frac{Z_{\alpha}}{n_{i} e} F_{e i \|}
$$

is the effective parallel electric field; $F_{e i \|}$ is the electron-ion parallel friction and

$$
\left.\frac{\partial}{\partial \psi} f_{\alpha 0} \equiv \frac{\partial}{\partial \psi} f_{\alpha 0}\right|_{v}=\left(\frac{\partial}{\partial \psi} \ln S \tau_{s}-\frac{v_{c}^{3}}{v^{3}+v_{c}^{3}} \frac{\partial}{\partial \psi} \ln v_{c}^{3}\right) f_{\alpha 0} .
$$

It is now apparent that $f_{\alpha 1}$ is independent of the $E \times B$ drift. In addition, all the terms involving ion flow are $\frac{T_{i}}{E_{\alpha}}$ smaller than the $\alpha$ particle diamagnetic drift terms. This justifies the adequacy of the results in Ref. 4 and rigorously proves that the neoclassical $\alpha$ particle radial fluxes do not explicitly depend on $E_{r}$. 
Now, $V_{\alpha i \|}$ can be calculated from

$$
n_{\alpha} V_{\alpha i \|}=\int d \mathbf{v} v_{\|} f_{\alpha 1}=\frac{\pi}{h} \sum_{\sigma} \sigma \int_{0}^{v_{0}} v^{3} d v \int_{0}^{h} d \lambda f_{\alpha 1}
$$

which yields

$$
\begin{aligned}
\left\langle n_{\alpha} \frac{V_{\alpha i \|}}{h}\right\rangle & =\left(1-\left\langle\frac{1}{h^{2}}\right\rangle \sum_{n=1}^{\infty} \frac{\gamma_{n}}{\kappa_{n}}\right)\left(U_{1}-n_{\alpha}\left\langle\frac{V_{\| i}^{*}}{h}\right\rangle\right) \\
& -\left\langle\frac{1}{h^{2}}\right\rangle \sum_{n=1}^{\infty}\left[\gamma_{n}\left(1-\frac{1}{\kappa_{n}}\right)\left(U_{2 n}-N_{2 n}\left\langle\frac{V_{\| i}^{*}}{h}\right\rangle\right)\right] \\
& +N_{3} \frac{Z_{\alpha} e \tau_{s}}{m_{\alpha}}\left\langle\frac{E_{\|}^{*}}{h}\right\rangle .
\end{aligned}
$$

Here, $\sigma \equiv v_{\|} /\left|v_{\|}\right|, \gamma_{n}$ is defined in Eq. (17) of Ref. 4,

$$
U_{1} \equiv \int d \mathbf{v} \frac{v^{2}}{3}\left[-\frac{I}{\Omega_{0}} \frac{\partial}{\partial \psi} f_{\alpha 0}\right] \equiv-\frac{I}{m_{\alpha} \Omega_{0}} \frac{\partial}{\partial \psi} P_{\alpha}=S \tau_{s} v_{0} \sum_{\ell=1}^{2} L_{\ell} X_{\ell}
$$

is the $\alpha$ diamagnetic flow,

$$
\begin{aligned}
& U_{2 n} \equiv \int d \mathbf{v} \frac{v^{2}}{3} H_{n}(v)\left(\frac{-I}{\Omega_{0}} \frac{\partial}{\partial \psi} f_{\alpha 0}\right), \\
& H_{n}(v) \equiv \frac{4}{v^{4}} \int_{0}^{v} d u \quad u^{3}\left(\frac{u^{3}\left(v^{3}+v_{c}^{3}\right)}{v^{3}\left(u^{3}+v_{c}^{3}\right)}\right)^{\frac{Q_{p} \kappa_{n}}{3}} \\
& N_{2 n} \equiv \int d \mathbf{v}\left[H_{n}(v)+\frac{v}{3} \frac{\partial}{\partial v} H_{n}(v)\right] f_{\alpha 0}, \\
& N_{3} \equiv \sum_{n=1}^{\infty} \gamma_{n} \int d \mathbf{v}\left(G_{n}(v)+\frac{v}{3} \frac{\partial}{\partial v} G_{n}(v)\right) f_{\alpha 0} \\
& G_{n}(v) \equiv \frac{1}{v} \int_{0}^{u} d u\left(\frac{u^{3}\left(v^{3}+v_{c}^{3}\right)}{v^{3}\left(u^{3}+v_{c}^{3}\right)}\right)^{\frac{Q_{p^{\alpha_{n}}}}{3}} \frac{u^{3}}{u^{3}+v_{c}^{3}} \\
& L_{\ell} \equiv \frac{1}{3 \chi_{0}^{3(\ell-1)}} \int_{0}^{1} d x \frac{x^{4}}{\left(x^{3}+\chi_{0}^{-3}\right)^{\ell}} \\
& X_{1} \equiv-\frac{I v_{0}}{\Omega_{0}} \frac{\partial}{\partial \psi}\left(\ln S \tau_{s}\right) \\
& X_{2} \equiv-\frac{I v_{0}}{\Omega_{0}} \frac{\partial}{\partial \psi}\left(\ln v_{c}^{3}\right)
\end{aligned}
$$


and $\chi_{0} \equiv \frac{v_{0}}{v_{c}}$ is the ratio of $\alpha$ birth to critical velocity.

Here, it is important to note some properties of $\gamma_{n}, \kappa_{n}$, and $f_{c}$, the effective fraction of circulating particles, ${ }^{5}$ i.e,

$$
\sum_{n=1}^{\infty} \frac{\gamma_{n}}{\kappa_{n}}=\frac{3}{4} \int_{0}^{\lambda_{c}} d \lambda \frac{\lambda}{\langle|\xi|\rangle} \equiv \frac{f_{c}^{p}}{\left\langle h^{-2}\right\rangle}
$$

which corresponds to the "pitch angle scattering" dominant solution of the $\alpha$ drift kinetic equation.

$$
\sum_{n=1}^{\infty} \gamma_{n}=-\frac{3}{4} \int_{0}^{\lambda_{c}} d \lambda\left(\frac{\partial\langle|\xi|\rangle}{\partial \lambda}\right)^{-1} \equiv \frac{f_{c}^{d}}{\left\langle h^{-2}\right\rangle}
$$

corresponds to the "drag" dominant case. $\xi \equiv \frac{v_{\|}}{v}$ is a pitch angle variable.

In addition, $H_{n}(v)$ and $G_{n}(v)$ have the following properties:

$$
H_{n}(v), G_{n}(v) \begin{cases}<1 & \text { for } Q_{p} \kappa_{n}>0 \\ =1 & \text { for } Q_{p} \kappa_{n}=0 \\ \sim O\left(\frac{1}{Q_{p} \kappa_{n}}\right) & \text { for } Q_{p} \kappa_{n} \gg 1\end{cases}
$$

Also, using the fact that $\kappa_{n}>2 n^{2}-n$ and typically $Q_{p} \sim 1$, one expects a rapid convergence of $H_{n}(v)$ and $G_{n}(v)$ with $n$. This implies that one needs only the first few eigenfunctions (e.g. $n=1 \rightarrow 5$ ) to accurately evaluate the second and third terms in Eq. (14).

Furthermore, when (i) $Q_{p} \gg 1$, (i.e. pitch angle scattering dominant), only the first term in Eq. (14) survives; and when (ii) $Q_{p} \ll 1$, Eq. (14) reduces to

$$
\begin{aligned}
\left\langle n_{\alpha} \frac{V_{\alpha i \|}}{h}\right\rangle & \simeq\left(1-\left\langle\frac{1}{h^{2}}\right\rangle \sum_{n=1}^{\infty} \gamma_{n}\right)\left(U_{1}-n_{\alpha}\left\langle\frac{V_{\| i}^{*}}{h}\right\rangle\right) \\
& +\left(\sum_{n=1}^{\infty} \gamma_{n}\right) n_{\alpha} \frac{Z_{\alpha} e \tau_{s}}{m_{\alpha}}\left\langle\frac{E_{\|}^{*}}{h}\right\rangle
\end{aligned}
$$

as is expected (also cf. Eq. (22)).

Thus, the $\alpha$ dynamics has been solved by including the effects of ion flow and the effective parallel electric field. However, these additional effects are insignificant quantitatively because (i) they are $O\left(\frac{T_{i}}{E_{\alpha}}\right)$ smaller than the $\alpha$ diamagnetic terms, and (ii) they 
contribute only $O\left(\frac{n_{\alpha} Z_{\alpha}^{2}}{n_{e}}\right)$ corrections to the conventional bootstrap current (as will be shown in the next section). One can thus simplify Eq. (14) by omitting these effects, and we obtain

$$
\left\langle n_{\alpha} \frac{V_{\alpha i \|}}{h}\right\rangle=f_{t}^{p} U_{1}-\left\langle\frac{1}{h^{2}}\right\rangle \sum_{n=1}^{\infty}\left(\gamma_{n}\left(1-\frac{1}{\kappa_{n}}\right) U_{2 n}\right),
$$

where $f_{t}^{p} \equiv 1-f_{c}^{p}$ corresponds to the fraction of trapped particles where $p$ denotes the pitch angle dominated solution, see Eq. (21).

Furthermore, although the second term of Eq. (24) can be evaluated using a few numerical eigenfunctions, it is highly desirable to write it in a simpler analytic form. Using the asymptotic behavior of $U_{2 n}$ at $Q_{p} \rightarrow 0, Q_{p} \gg 1, \chi_{0} \rightarrow 0, \chi_{0} \gg 1$, a Padé form is obtained in Eq. (B6) of Appendix B. Equation (24) can thus be written as

$$
\left\langle\frac{n_{\alpha} V_{\alpha i \|}}{h}\right\rangle=S \tau_{s} v_{0} \sum_{\ell=1}^{2}\left(f_{t}^{d}+\frac{Q_{p} L_{\ell}}{Q_{p} L_{\ell}+4 f_{c}^{p} L_{\ell-1}}\left(f_{t}^{p}-f_{t}^{d}\right)\right) L_{\ell} X_{\ell}
$$

The first term in the bracket is clearly due to the drag and the second term is due to the pitch angle scattering effects. Note that for $\epsilon \ll 1, f_{t}^{d} \simeq 1.6 \epsilon^{3 / 2}$, and $f_{t}^{p} \simeq 1.46 \epsilon^{1 / 2}$. Since $f_{t}^{d} / f_{t}^{p} \simeq \epsilon$, it does not require a very large $Q_{p}$ to make the pitch angle scattering effects important.

Furthermore, the Padé approximations of $f_{c}^{d}, f_{c}^{p}$ for arbitrary $\epsilon$ are obtained in Eq. (B9). Note that, as shown in Fig. (1), Eq. (B9) is in excellent agreement with the exact numerical calculations. It is also worth mentioning that by using Eq. (B9), the simple analytic Padé form Eq. (25) has been compared with numerical results evaluated from Eq. (24). Remarkably, over wide ranges of $\epsilon, \chi_{0}$, and $Q_{p}$, the difference is always within $10 \%$. This actually implies that the error of Eq. (25) is much less than $10 \%$ (cf. Appendix B). 


\section{CALCULATION OF $V_{e i}$}

First, using the facts that $\frac{m_{a}}{m_{e}} \gg 1$ and $\frac{V_{a}}{v_{\text {the }}} \ll 1$, one can obtain an approximate $e-\alpha$ collision operator in the ion rest frame as

$$
C_{e \alpha}=\frac{3}{4} \sqrt{\pi} \frac{v_{t h e}^{3}}{\tau_{e}} \frac{n_{\alpha} Z_{\alpha}^{2}}{n_{e} Z_{e f f}}\left[\frac{\partial}{\partial \mathrm{v}} \cdot \stackrel{\leftrightarrow}{\mathrm{U}} \cdot \frac{\partial}{\partial \mathrm{v}} f_{e}+\frac{2 \mathrm{~V}_{\alpha i} \cdot \mathrm{v}}{v_{t h e}^{2} v^{3}} f_{e M}\right]
$$

That is,

$$
C_{e \alpha}+C_{e i}=\frac{3}{4} \sqrt{\pi} \frac{v_{t h e}^{3}}{\tau_{e}}\left[\left(1+\frac{n_{\alpha} Z_{\alpha}^{2}}{n_{e} Z_{e f f}}\right) \frac{\partial}{\partial \mathrm{v}} \cdot \stackrel{\leftrightarrow}{\mathrm{U}} \cdot \frac{\partial}{\partial \mathrm{v}} f_{e}+\frac{n_{\alpha} Z_{\alpha}^{2}}{n_{e} Z_{\text {eff }}} \frac{2 \mathrm{v}_{\alpha i} \cdot \mathrm{v}}{v_{t h e}^{2} v^{3}} f_{e M}\right] .
$$

Here, $\overleftrightarrow{\mathbf{U}} \equiv \frac{v^{2} \overleftrightarrow{\mathbf{I}}-\mathbf{v v}}{v^{3}}, Z_{e f f} \equiv \sum_{i} n_{i} \frac{Z_{i}^{2}}{n_{e}}$ and the equality $\frac{m_{e} Z_{\alpha}^{2}}{m_{\alpha} \tau_{e} Z_{e f f}}=\frac{1}{\tau_{e}}$ has been used.

Note that the first term in Eq. (26) corresponds to pitch angle scattering and that the second term is a momentum input to the electrons due to $\mathrm{V}_{\alpha i}$. Also note that the $\alpha$ contribution to pitch angle scattering is insignificant because it simply replaces $\frac{1}{r_{e}}$ with $\frac{1}{\tau_{e}}\left(1+\frac{n_{\alpha} Z_{\alpha}^{2}}{n_{e} Z_{e f f}}\right)$ and $\frac{n_{\alpha} Z_{\alpha}^{2}}{n_{e} Z_{e f f}} \ll 1$. On the other hand, the momentum input term involving $V_{\alpha i}$ is $O\left(\frac{\beta_{\alpha} Z_{\alpha}^{2}}{\beta_{i} Z_{\text {eff }}}\right)$ compared with the first term in Eq. (26), and thus can be significant. Furthermore, since $\frac{F_{e \alpha}}{F_{e i}} \sim O\left(\frac{\beta_{\alpha} Z_{\alpha}^{2}}{\beta_{i} Z_{e f f}}\right)$, which can be at most of order one in a typical fusion device, one can conclude that ion and impurity dynamics will hardly be modified by alphas because $\left\langle\mathbf{B} \cdot \boldsymbol{\nabla} \cdot \overleftrightarrow{\boldsymbol{\Pi}}_{i}\right\rangle \gg\left\langle B F_{\text {ei } \|}\right\rangle \geq\left\langle B F_{\text {ea } \|}\right\rangle \gtrsim\left\langle B F_{i \alpha \|}\right\rangle$.

To solve the electron dynamics the moment approach as developed in Ref. 5 will be adopted in order to retain generality with respect to the electron collisionality. In addition, because the parallel flow viscosity and heat viscosity solely depend on the poloidal particle flow, heat flow and collisionality of the individual species, both viscous forces do not change their form in the presence of $\alpha$ particles, except for a trivial increase of $\nu_{e i}$ (or $Z_{\text {eff }}$ ), i.e., $\nu_{e i}=\nu_{e i}^{(0)}\left(1+\frac{n_{\alpha} Z_{\alpha}^{2}}{n_{e} Z_{e f f}^{(0)}}\right)$. Therefore, the viscous forces obtained in Ref. 5 can be used here without modification.

The moment equations to be solved are the electron parallel momentum equation

$$
\left\langle\mathbf{B} \cdot \boldsymbol{\nabla} \cdot \overleftrightarrow{\mathbf{I}}_{\mathrm{e}}\right\rangle=-n_{e} e\left\langle E_{\|} B\right\rangle+\left\langle F_{e \|} B\right\rangle
$$


and the electron parallel heat momentum equation

$$
\left\langle\mathbf{B} \cdot \boldsymbol{\nabla} \cdot \overleftrightarrow{\boldsymbol{\Theta}}_{\mathrm{e}}\right\rangle=\left\langle F_{\mathrm{e} \|}^{(2)} B\right\rangle
$$

where

$$
\begin{aligned}
\overleftrightarrow{\Theta}_{e} & \equiv \int d \mathbf{v} \frac{m_{e}}{2}\left(v^{2}-\frac{5}{2} v_{t h e}^{2}\right)\left(v \mathbf{v}-\frac{v^{2}}{3} \overleftrightarrow{\mathbf{I}}\right) f_{e}, \\
\mathbf{F}_{e}^{(2)} & \equiv \int d \mathbf{v} \frac{m_{e} \mathbf{v}}{2}\left(v^{2}-\frac{5}{2} v_{t h e}^{2}\right) C_{e}\left(f_{e}\right) .
\end{aligned}
$$

Using the two-moment approximation ${ }^{5,6}$ and Eq. (26), the frictional moments can be written as

$$
\begin{aligned}
\mathbf{F}_{e} & =m_{e} n_{e} \nu_{e i}\left(\mathrm{~V}_{*}+\frac{3}{5} \frac{\mathbf{q}_{e}}{n_{e} T_{e}}\right) \\
\mathbf{F}_{e}^{(2)} & =-\frac{3}{2} n_{e} T_{e} \nu_{e i}\left(\mathrm{~V}_{*}+\frac{8}{15}\left(\nu_{e e}+\frac{13}{8} \nu_{e i}\right) \frac{\mathbf{q}_{e}}{n_{e} T_{e}}\right)
\end{aligned}
$$

where $\mathbf{V}_{*} \equiv \mathbf{V}_{i e}+\frac{n_{\alpha} Z_{\alpha}^{2}}{n_{e} Z_{e f f}} \mathbf{V}_{\alpha i}$. The viscous forces ${ }^{5}$ are

$$
\begin{aligned}
& \left\langle\mathbf{B} \cdot \boldsymbol{\nabla} \cdot \stackrel{\leftrightarrow}{\mathbf{\Pi}}_{e}\right\rangle=m_{e} n_{e}\left\langle B^{2}\right\rangle\left(\mu_{1} V_{p}+\frac{2}{5} \mu_{2} q_{p}\right) \\
& \left\langle\mathbf{B} \cdot \boldsymbol{\nabla} \cdot \overleftrightarrow{\boldsymbol{\Theta}}_{e}\right\rangle=n_{e} T_{e}\left\langle B^{2}\right\rangle\left(\mu_{2} V_{p}+\frac{2}{5} \mu_{3} q_{p}\right)
\end{aligned}
$$

Here,

$$
\begin{aligned}
\mathbf{V}_{e} & =V_{p} \mathbf{B}+\omega_{1} R^{2} \nabla \phi \\
\mathbf{q}_{e} & =n_{e} T_{e}\left(q_{p} \mathrm{~B}+\omega_{2} R^{2} \nabla \phi\right) \\
\omega_{1} & =\frac{B}{m_{e} n_{e} \Omega_{e}}\left[\frac{\partial}{\partial \psi} p_{e}-n_{e} e \frac{\partial}{\partial \psi} \Phi\right] \\
\omega_{2} & =\frac{5}{2} \frac{B}{m_{e} \Omega_{e}} \frac{\partial}{\partial \psi} T_{e}
\end{aligned}
$$

and fitted Padé forms of $\mu_{1}, \mu_{2}, \mu_{3}$ for all collisionality regimes can be found in Ref. 5 . Note that in the banana regime

$$
\mu_{1} \simeq \frac{f_{t}^{p}}{f_{c}^{p}}\left(\nu_{e i}+0.754 \nu_{e e}\right)
$$




$$
\begin{aligned}
\mu_{2} & \simeq-\frac{f_{t}^{p}}{f_{c}^{p}}\left(1.5 \nu_{e i}+0.884 \nu_{e e}\right) \\
\mu_{3} & \simeq \frac{f_{t}^{p}}{f_{c}^{p}}\left(3.25 \nu_{e i}+1.94 \nu_{e e}\right) .
\end{aligned}
$$

Concerning $\mathbf{V}_{\alpha i}$, it is now clear that the terms involving $\left\langle\frac{V_{\| i}}{h}\right\rangle$ and $\left\langle\frac{E_{\|}^{*}}{h}\right\rangle$ in Eq. (14) are $O\left(\frac{n_{\alpha} Z_{\alpha}^{2}}{n_{e} Z_{\text {eff }}}\right)$ compared with $\left\langle\frac{V_{\text {ie\|l}}}{h}\right\rangle$ terms in Eqs. (29) and (30), and $\left\langle E_{\|} B\right\rangle$ terms in Eq. (27) respectively. Equations (27)-(36) thus yield

$$
\left\langle V_{i e \|} B\right\rangle=\left\langle V_{i e \|}^{(0)} B\right\rangle\left(1+O\left(\frac{n_{\alpha} Z_{\alpha}^{2}}{n_{e} Z_{e f f}}\right)\right)-\left(1-F_{\mu}^{e}\right) \frac{n_{\alpha} Z_{\alpha}^{2}}{n_{e} Z_{e f f}}\left\langle\mathbf{V}_{\alpha i \|} B\right\rangle,
$$

where

$$
F_{\mu}^{e} \equiv \frac{\mu_{1}\left[\mu_{3}+2\left(\nu_{e e}+\frac{13}{8} \nu_{e i}\right)\right]-\mu_{2}\left(\mu_{2}-\frac{3}{2} \nu_{e i}\right)}{\left(\mu_{1}+\nu_{e i}\right)\left[\mu_{3}+2\left(\nu_{e e}+\frac{13}{8} \nu_{e i}\right)\right]-\left(\mu_{2}-\frac{3}{2} \nu_{e i}\right)^{2}}
$$

is the effective fraction of trapped electrons; and $\left\langle V_{\alpha i \|} B\right\rangle=B_{0}\left\langle\frac{V_{\alpha i \| l}}{h}\right\rangle$ can be evaluated using Eqs. (24) and (25). Note that the superscript (0) in Eq. (37) refers to the conventional results without the contribution from $\alpha$ particles. Also, from the fact that $f_{t}^{p} \ll f_{c}^{p}$ at large aspect ratio $(\epsilon \ll 1)$, one has $\mu_{j} \ll \nu_{e i}$, and $F_{\mu}^{e} \rightarrow 0$. Consequently, for $\epsilon \ll 1$,

$$
\left\langle V_{i e \|} B\right\rangle=\left\langle V_{i e \|}^{(0)} B\right\rangle\left(1+O\left(\frac{n_{\alpha} Z_{\alpha}^{2}}{n_{e} Z_{e f f}}\right)\right)-\frac{n_{\alpha} Z_{\alpha}^{2}}{n_{e} Z_{e f f}}\left\langle V_{\alpha i \|} B\right\rangle
$$

This can also be understood from the fact that for $\epsilon \ll 1$, the poloidal flow $V_{\boldsymbol{p}}$ of the electrons is solely driven by the ion poloidal flow via electron-ion friction; and the poloidal heat flow $q_{p}$ is solely determined by the electron diamagnetic heat flow. This implies that the total electron friction is unchanged by the alphas. Therefore, $\left\langle V_{i e \|} B\right\rangle$ will be reduced simply according to the momentum input from $\left(\left.\right|_{\alpha i \|} B\right\rangle$ via electron- $\alpha$ friction (see also Eq. (29)). This also implies that, for $\epsilon \ll 1$, the electron radial flux is not changed by $\alpha$ particles. However, for generality, Eq. (37) should be used to include the finite $\epsilon$ effects. 


\section{BOOTSTRAP CURRENT}

It is now straightforward to calculate the total parallel current from Eqs. (1) and (37). This yields

$$
\left\langle J_{\|} B\right\rangle=\left\langle J_{\|}^{(0)} B\right\rangle\left(1+O\left(\frac{n_{\alpha} Z_{\alpha}^{2}}{n_{e} Z_{e f f}}\right)\right)+n_{\alpha} Z_{\alpha} e\left(1-\frac{Z_{\alpha}}{Z_{e f f}}\left(1-F_{\mu}^{e}\right)\right)\left\langle V_{\alpha i \|} B\right\rangle
$$

where $\left\langle V_{\alpha i \|} B\right\rangle$ is given in Eqs. (24) and (25). Eqs. (24), (25) and (39) thus yield the bootstrap current produced by alphas as

$$
J_{b s}^{\alpha}=\left(1-\frac{Z_{\alpha}}{Z_{e f f}}\left(1-F_{\mu}^{e}\right)\right) F_{\mu}^{\alpha}\left(\frac{-c I}{B_{0}} \frac{\partial}{\partial \psi} P_{\alpha}\right)
$$

Here, the term involving $\frac{Z_{\alpha}}{Z_{e f f}}$ in Eq. (40) corresponds to the well known electron screening effects, 5,7 and

$$
F_{\mu}^{\alpha} \equiv f_{t}^{p}-\frac{\left\langle h^{-2}\right\rangle \sum_{n=1}^{\infty} \gamma_{n}\left(1-\frac{1}{\kappa_{n}}\right) U_{2 n}}{U_{1}} \simeq f_{t}^{d}+\frac{\sum_{\ell=1}^{2}\left(\frac{Q_{p} L_{\ell}}{Q_{p} L_{\ell}+4 f_{c}^{p} L_{\ell-1}}\left(f_{t}^{p}-f_{t}^{d}\right)\right) L_{\ell} X_{\ell}}{\sum_{\ell=1}^{2} L_{\ell} X_{\ell}}
$$

is the effective fraction of trapped alphas.

Note that the second term in Eq. (41) involving $Q_{p}$ is due to the effects of pitch angle scattering. When $Q_{p} \rightarrow 0$ or $v_{0} / v_{c} \gg 1$ (in which case $L_{\ell} / L_{\ell+1} \gg 1$ ), this term becomes negligible and the result reduces to the drag-only result $f_{t}^{d}$. However, since $Q_{p}$ is always $\sim 1$, and $f_{t}^{d} / f_{t}^{p} \simeq \epsilon$, it is not difficult to make the second term in Eq. (41) significant. In Fig. (2), results for $F_{\mu}^{\alpha}$ are shown for different $v_{0}$. The asymptotic case $E_{0}=\infty$ refers to the drag-only result. It is seen that $F_{\mu}^{\alpha}$ increases with decreasing $v_{0}$. The reason all expressions reduce to the drag-only result at $r / a=1$ is that $v_{c}=0$ at this point. This is because the temperature profile is taken to be $T_{0}\left(1-r^{2} / a^{2}\right)^{\nu_{T}}$.

In order to evaluate the bootstrap current quantitatively, it is essential to self-consistently solve for the equilibrium after incorporating $J_{b s}(\psi)$ into the parallel Ampere's law. In this work, for simplicity, we assume circular geometry, i.e,

$$
\frac{I}{B_{0}} \frac{\partial}{\partial \psi} \simeq \frac{1}{B_{p}} \frac{\partial}{\partial r}
$$


The parallel Ampere's law thus becomes

$$
\frac{1}{r} \frac{\partial}{\partial r} r B_{p}=\frac{4 \pi}{c}\left(J_{O H}+J_{b s}^{(0)}+J_{b s}^{\alpha}\right)
$$

Here,

$$
J_{O H}=F_{\mu \sigma}^{e} \sigma_{S p i t z e r}\left\langle E_{\|} / h\right\rangle
$$

is the ohmic current;

$$
J_{b s}^{(0)}=\left(\frac{-c I}{B_{0}}\right)\left[F_{\mu}^{e} \frac{\partial}{\partial \psi}\left(P_{e}+P_{i}\right)-F_{\mu}^{e} F_{\mu T^{i}}^{n_{i}} \frac{\partial}{\partial \psi} T_{i}-F_{\mu T^{e}}^{n_{e}} \frac{\partial}{\partial r} T_{e}\right]
$$

is the background bootstrap current;

$$
F_{\mu \sigma}^{e}=\frac{\left(\mu_{3}+2\left(\nu_{e e}+\frac{13}{8} \nu_{e i}\right)\right) \nu_{e i}}{\left(\mu_{1}+\nu_{e i}\right)\left[\mu_{3}+2\left(\nu_{e e}+\frac{13}{8} \nu_{e i}\right)\right]-\left(\mu_{2}-\frac{3}{2} \nu_{e i}\right)^{2}}
$$

describes the neoclassical correction to Spitzer conductivity, ${ }^{8}$ and

$$
F_{\mu T}^{e}=\frac{-2 \mu_{2}\left(\nu_{e e}+\frac{13}{8} \nu_{e i}\right)-1.5 \mu_{3} \nu_{e i}}{\left(\mu_{1}+\nu_{e i}\right)\left[\mu_{3}+2\left(\nu_{e e}+\frac{13}{8} \nu_{e i}\right)\right]-\left(\mu_{2}-\frac{3}{2} \nu_{e i}\right)^{2}}
$$

$F_{\mu T}^{i}$ is obtained by letting $\nu_{e i} \rightarrow 0$ and replacing $\nu_{e e} \rightarrow \nu_{i i}$ in Eqs. (46) and (36). For ions in the banana regime with $\epsilon \ll 1, F_{\mu T}^{i} \simeq 1.17$. Note that terms involving $F_{\mu T}^{i, e}$ are due to the thermal friction force.

Equations $(40)-(46)$ are solved numerically by adopting ITER parameters ${ }^{1}$ at the steady state phase: $\left\langle n_{e}\right\rangle=0.64 \times 10^{20} \mathrm{~m}^{-3},\left\langle T_{e}\right\rangle=20 \mathrm{KeV}, I_{p}=19 \mathrm{MA}, \kappa=2, \beta_{p}=1.1$, $a=2.15 \mathrm{~m}, R_{0}=6 \mathrm{~m}$. Also, the radial profile is assumed to be $n_{e} \propto\left(1-r^{2} / a^{2}\right)^{\nu_{n}}$, $T_{e} \propto\left(1-r^{2} / a^{2}\right)^{\nu_{T}}$ with $\nu_{n}=0.5, \nu_{T}=0.75$, and the inductive parallel electric field $\left\langle E_{\|} / h\right\rangle$ is chosen so that the total plasma current including the bootstrap currents reaches $I_{p} \simeq 19 \mathrm{MA}$. For $F_{\mu}^{\alpha}, f_{t}^{d}$, and $f_{t}^{p}$, the Padé forms given in Eqs. (41) and (B9) are used. Also, both electron and ion species are assumed to be in the banana regime throughout the plasma. The effect of elongation is considered by replacing the plasma minor radius by $a \sqrt{\kappa}$.

First, with $Z_{\text {eff }}=1.5$, the resulting poloidal magnetic field and bootstrap current densities are shown in Figs. (3)-(5). One notices the slightly decreased $B_{p}$ due to $J_{b s}^{\alpha}$. 
The alpha induced bootstrap current amounts to $\simeq 0.385 \mathrm{MA}$, which is $\simeq 7.2 \%$ of the background bootstrap current.

Varying $Z_{\text {eff }}$, one finds a strong dependence of $J_{b s}^{\alpha}$ on $Z_{\text {eff }}$. In fact, the effects of $Z_{\text {eff }}$ on $J_{b s}^{\alpha}$ are threefold:

(i) The electron screening effect decreases with increasing $Z_{\text {eff }}$. Indeed, for $Z_{\text {eff }}<Z_{\alpha}$, at $\epsilon \ll 1, F_{\mu}^{e}$ becomes negligible and $J_{b s}^{\alpha}$ becomes negative.

(ii) Alpha pitch angle scattering effects increase with $Z_{\text {eff }}$ (because $Q_{p} \propto Z_{\text {eff }}$ ). That is, $F_{\mu}^{\alpha}$ increases with $Z_{\text {eff }}$.

(iii) However, the $\alpha$ pressure decreases with $Z_{\text {eff }}$ due to fuel dilution. Actually, when $Z_{\text {eff }}(r)$ is highly peaked near the axis, both $P_{t h}(r)$ and $P_{\alpha}(r)$ can become flat or even hollow; and the bootstrap current density $J_{b s}$ can be significantly reduced.

Table 1 shows the final currents for two cases where the $Z_{\text {eff }}$ profile is flat; one where $Z_{\text {eff }}=1.5 ;$ the other where $Z_{\text {eff }}=2.2$. The ohmic seed current is kept fixed at $13 \mathrm{MA}$. For $Z_{\text {eff }}=1.5$, the background bootstrap current $\simeq 30 \%$ of the total current. The calculated alpha induced bootstrap current reaches $\simeq 7.2 \%$ of the conventional bootstrap current. When $Z_{\text {eff }}$ is increased to $Z_{\text {eff }}=2.2$, the total current decreases slightly by about $2 \%$. On the other hand, the conventional bootstrap current decreases by $5 \%$ while the alpha bootstrap current decreases by $21 \%$. Although a larger $Z_{\text {eff }}$ has the effect of diminishing the temperature screening term in the alpha bootstrap current (see Eq. (40)), and increasing the alpha pitch angle scattering, increased fuel dilution has a dominant effect. Specifically, when $Z_{\text {eff }}$ is increased, causing more fuel dilution, the alpha and thermal pressure decreases resulting in less bootstrap current. Since the dilution effect is squared in the alpha pressure while occurring only linearly in the thermal pressure, the effect on the alpha bootstrap current is pronounced.

The effect of having a peaked $Z_{\text {eff }}$ profile was also investigated. Here we let $Z_{\text {eff }}(r)=$ $0.7 \exp \left[-16 r^{2} / a^{2}\right]+1.5$ making $Z_{\text {eff }}=2.2$ at the the center and 1.5 near the edge. Again, the dominant $Z_{\text {eff }}$ effect is fuel dilution. The resulting currents for this case are listed in Table 1 and can be seen to be quite similar to the flat $Z_{\text {eff }}=1.5$ case. This is because, in the peaked case, $Z_{\text {eff }}$ rises significantly above 1.5 only near the center where there is 
little bootstrap current anyway. Note that both the background and the alpha induced bootstrap current densities become slightly negative near the center. The reason is that the fuel dilution gradient (specifically $\left.d\left(Z_{\text {eff }}\right) / d r\right)$ ) has a diminishing effect on the negative thermal and alpha pressure gradients, and at some small value of $r / a$ causes the bootstrap current to change direction.

On the other hand, with the fuel dilution effects switched off, the ratio of alpha to background bootstrap current density is given in Fig. (6), for both the flat $Z_{\text {eff }}=1.5$ and the centrally peaked $Z_{\text {eff }}(r)$. It is shown that the alpha induced bootstrap current density increases with $Z_{\text {eff }}$ due to the increasing alpha pitch angle scattering and decreasing electron current screening.

Furthermore, with $Z_{e f f}=1.5$, by artificially switching off the effects of fuel dilution and pitch angle scattering individually, as shown in Table 2, the significance of these effects can be clearly observed. It is found that the effects of pitch angle scattering enhance $I_{b s}^{\alpha}$ only by $30 \%$ while the fuel dilution can reduce $I_{b s}^{\alpha}$ by $20 \%$. The reason is that the pitch angle scattering is most important near the center where the bootstrap current density is small.

Equation (40) is good for arbitrary $v_{0} / v_{c}$ and $\epsilon$ and is valid for evaluating the bootstrap current induced by any hot species containing sufficient isotropic pressure. One concludes that the enhancement of the bootstrap current contribution from energetic ions including pitch angle scattering will drastically increase with smaller $v_{0}$.

\section{v. CONCLUSION}

The bootstrap current induced by fusion born $\alpha$ particles has been calculated for general electron collisionality. Our result retains the effects of both $\alpha$ pitch angle scattering and slowing down drag for arbitrary aspect ratio. The results are presented in Eqs. (40) and (41), in both forms: a summation over a rapidly converging series $\sum_{n=1}^{\infty} \gamma_{n}\left(1-\frac{1}{\kappa_{n}}\right) U_{2 n}$ and a simple Padé approximation good for arbitrary $\frac{v_{0}}{v_{c}}$ and $\epsilon$. Convenient Padé forms of the effective fraction of circulating particles $f_{c}^{d}$ and $f_{c}^{p}$ are presented in Eq. (B9). It has 
also been shown that the radial electric field $E_{r}$ does not play a role in $\alpha$ particle transport [cf. Eq. (A5)].

The effects of $Z_{\text {eff }}$ on $J_{b s}^{\alpha}$ are significant because when $Z_{\text {eff }}$ increases the electron screening effect decreases and the $\alpha$ pitch angle scattering effect increases but the $\alpha$ pressure decreases due to fuel dilution which has been found to be a strong effect. Actually, the reason that $\alpha$ pitch angle scattering enhances $I_{b s}^{\alpha}$ only by $30 \%$ is due to the effects of electron screening and strong fuel dilution. When $Z_{e f f}\left(1-F_{\mu}^{e}\right)<Z_{\alpha}, J_{b s}^{\alpha} / J_{b s}^{(0)}$ can become negative.

The theoretical results are evaluated quantitatively by using the ITER parameters and numerically solving the parallel Ampere's law including the bootstrap current terms $\propto B_{p}^{-1}$ for the self-consistent $B_{p}(r)$. It is found that the bootstrap current produced by alpha particles reaches $\simeq 0.385 \mathrm{MA}$ and is about $7.2 \%$ of the conventional bootstrap current.

The results given in Eqs. (40) and (41) are valid for evaluating the bootstrap current induced by any hot isotropic species. For an anisotropic hot species generated by neutral beam injection or $\mathrm{rf}$ heating, the induced bootstrap current can be calculated by straightforwardly extending the present approach to also include the eigenfunctions even in $v_{\|}$ (work in progress). 


\section{Acknowledgment}

This work was supported by the US Department of Energy under Grant DE-FG0291ER-54109. 


\section{Appendix A}

\section{Derivation of $f_{\alpha}$}

Since the source term of fusion born alpha particles is isotropic in the ion rest frame, we treat the alpha kinetic equation in this frame. Starting from the kinetic equation, changing variable $\mathbf{v} \rightarrow \mathbf{v}-\mathbf{V}_{i}$ and then performing the gyro-average ${ }^{9}$ the $\alpha$ drift kinetic equation to first order in gyroradius becomes

$$
\begin{gathered}
v_{\|} \nabla_{\|} \bar{f}_{\alpha}+\mathbf{v}_{d} \cdot\left(\nabla f_{\alpha 0}+\mathbf{F}_{0} \frac{\partial}{\partial w} f_{\alpha 0}\right)+v_{\|} F_{\|}^{(1)} \frac{\partial}{\partial w} f_{\alpha 0}-\left[\left(\mathbf{b} \cdot \overleftrightarrow{\mathbf{w}}_{i} \cdot \mathbf{b}\right)\left(\frac{3 v_{\|}^{2}-v^{2}}{2}\right)\right. \\
\left.+\frac{2 v^{2}}{3} \nabla \cdot \mathbf{v}_{i}\right] \frac{\partial}{\partial w} f_{\alpha 0}=C_{\alpha}\left(\bar{f}_{\alpha}\right)+\frac{S}{4 \pi v^{2}} \delta\left(v-v_{0}\right) .
\end{gathered}
$$

Here, $f_{\alpha} \equiv f_{\alpha}(\lambda, w, \psi, \theta) ; \nabla$ is performed at constant $(\lambda, w)$;

$$
\mathbf{F} \equiv\left(\frac{m_{i} Z_{\alpha}}{m_{\alpha} Z_{i}}-1\right)\left(\frac{\partial}{\partial t} \mathbf{V}_{i}+\mathbf{V}_{i} \cdot \nabla \mathbf{V}_{i}\right)+\frac{Z_{\alpha}}{m_{\alpha} Z_{i} n_{i}}\left(\nabla p_{i}+\nabla \cdot \overleftrightarrow{\mathbf{M}}_{i}-\mathbf{R}_{i}\right)
$$

$\mathbf{v}_{d}=v_{\|} \mathbf{b} \times \nabla \frac{v_{\|}}{\Omega}$ is the drift velocity in the ion rest frame; and

$$
\overleftrightarrow{\mathrm{W}}_{i} \equiv \frac{1}{2}\left(\left(\nabla \mathrm{V}_{i}\right)+\left(\nabla \mathrm{V}_{i}\right)^{T}\right)-\frac{1}{3}\left(\nabla \cdot \mathrm{V}_{i}\right) \stackrel{\leftrightarrow}{\mathrm{I}}
$$

is the ion velocity strain.

Note that Eq. (A1) agrees with the results by Hazeltine and Ware ${ }^{9}$ except for the definition of the $\mathbf{F}$ force term. This is because the results in Ref. 9 are for the drift kinetic equation for a given species in its own rest frame. Furthermore, Eq. (A1) also agrees with Eq.(5.45) of Hinton and Hazeltine ${ }^{10}$ which is the electron drift kinetic equation in the ion rest frame.

From Eq. (A2) and $\mathbf{V}_{i}=K_{i} \mathrm{~B}+\omega_{i} R^{2} \nabla \phi$ one finds

$$
\mathbf{F}_{0}=\frac{Z_{\alpha}}{m_{\alpha} Z_{i} n_{i}} \nabla p_{i}, F_{\|}^{(1)}=\frac{Z_{\alpha} e}{m_{\alpha}} E_{\|}, \nabla \cdot \mathrm{v}_{i}=0, \mathrm{~b} \cdot \overleftrightarrow{\mathrm{w}}_{i} \cdot \mathrm{b}=K_{i} \mathbf{b} \cdot \nabla B
$$

Equations (A1) and (A4) thus yield

$$
v_{\|} \nabla_{\|}\left[\bar{f}_{\alpha}+\frac{I v_{\|}}{\Omega_{\alpha}} \frac{\partial}{\partial \psi} f_{\alpha 0}-v_{\|} V_{\| i}^{*} \frac{\partial}{\partial w} f_{\alpha 0}\right]+\frac{z_{\alpha} e}{m_{\alpha}} v_{\|} E_{\|} \frac{\partial}{\partial w} f_{\alpha 0}
$$




$$
=C_{\alpha}\left(\bar{f}_{\alpha}\right)+\frac{S}{4 \pi v^{2}} \delta\left(v-v_{0}\right)
$$

Let us now turn to the $\alpha$ collision operator in the ion rest frame. By using $\frac{v_{\alpha}}{v_{\text {the }}} \ll 1$, $\frac{m_{e}}{m_{\alpha}} \ll 1$, and Eq. (26), one finds

$$
C_{\alpha e}=\frac{1}{\tau_{s}} \frac{\partial}{\partial \mathbf{v}} \cdot\left(\mathbf{v} f_{\alpha}+\frac{T_{e}}{m_{\alpha}} \frac{\partial}{\partial \mathbf{v}} f_{\alpha}\right)+\frac{\tau_{e}}{\tau_{s} m_{e} n_{e}} \mathbf{F}_{e i} \cdot \frac{\partial}{\partial \mathbf{v}} f_{\alpha 0}
$$

Here, $F_{e i}$ is the electron-ion friction. Similarly, $C_{\alpha i}$ can be written as

$$
C_{\alpha i}=\frac{v_{b}^{3}}{2 \tau_{s}} \frac{\partial}{\partial \mathbf{v}} \cdot \overleftrightarrow{\mathrm{U}} \cdot \frac{\partial}{\partial \mathrm{v}} f_{\alpha}+\frac{v_{c}^{3}}{\tau_{s}} \frac{\partial}{\partial \mathbf{v}} \cdot \frac{\mathbf{v}}{v^{3}} f_{\alpha}
$$

Equations (A5) - (A7) thus lead to

$$
\begin{gathered}
v_{\|} \nabla_{\|}\left[\bar{f}_{\alpha}+\frac{I v_{\|}}{\Omega_{\alpha}} \frac{\partial}{\partial \psi} f_{\alpha 0}-v_{\|} V_{\| i}^{*} \frac{\partial}{\partial w} f_{\alpha 0}\right]+\frac{Z_{\alpha} e}{m_{\alpha}} v_{\|} E_{\|}^{*} \frac{\partial}{\partial w} f_{\alpha 0} \\
=\frac{1}{\tau_{s}}\left[\frac{\partial}{\partial v} \cdot\left(1+\frac{v_{c}^{3}}{v^{3}}\right) \mathbf{v} \bar{f}_{\alpha}+\frac{v_{b}^{3}}{2} \frac{\partial}{\partial v} \cdot \overleftrightarrow{\mathrm{U}} \cdot \frac{\partial}{\partial v} \bar{f}_{\alpha}\right]+\frac{S}{4 \pi v^{2}} \delta\left(v-v_{0}\right) .
\end{gathered}
$$

Then, by following the same procedure as in Ref. 4, one obtains Eqs. (2) and (3) with the governing equations for $V_{n j}$ :

$$
\begin{aligned}
& v_{b}^{3}\left(\frac{\partial}{\partial \psi} f_{\alpha 0}\right)\left(\sigma_{n}-\kappa_{n} V_{n 1}\right)=\frac{\partial}{\partial v}\left[\left(v^{3}+v_{c}^{3}\right) v\left(\frac{\partial}{\partial \psi} f_{\alpha 0}\right)\left(\sigma_{n}-V_{n 1}\right)\right] \\
& v_{b}^{3}\left(\frac{\partial}{\partial w} f_{\alpha 0}\right)\left(\sigma_{n}-\kappa_{n} V_{n 2}\right)=\frac{\partial}{\partial v}\left[\left(v^{3}+v_{c}^{3}\right)\left(\frac{\partial}{\partial v} f_{\alpha 0}\right)\left(\sigma_{n}-V_{n 2}\right)\right] \\
& v_{b}^{3}\left(\frac{\partial}{\partial w} f_{\alpha 0}\right) \kappa_{n} V_{n 3}+v^{3}\left(\frac{\partial f_{\alpha 0}}{\partial w}\right) \sigma_{n}=\frac{\partial}{\partial v}\left[\left(v^{3}+v_{c}^{3}\right)\left(\frac{\partial}{\partial v} f_{\alpha 0}\right) V_{n 3}\right]
\end{aligned}
$$

which leads to the solutions given in Eqs. (8)-(10). 


\section{Appendix B}

\section{Padé Approximation}

The right hand side of Eq. (24) is rigorously expressed by summing over a rapidly

converging series $\gamma_{n}\left(1-\frac{1}{\kappa_{n}}\right) U_{2 n}$ and can be evaluated quite accurately by using only a few eigenfunctions. However, it is highly desirable to get a simpler analytic form good for all $\chi_{0}, Q_{p}$, and $\epsilon$. Let's start with $U_{2 n}$, which can be written as

$$
U_{2 n}=S \tau_{s} v_{0} \sum_{\ell=1}^{2} D_{n \ell} X_{\ell}
$$

where

$$
D_{n \ell} \equiv \frac{1}{3 \chi_{0}^{3(\ell-1)}} \int_{0}^{1} d x \frac{x^{4}}{\left(x^{3}+\chi_{0}^{-3}\right)^{\ell}} H_{n}\left(v_{0} x\right) .
$$

From the asymptotic behaviors of $H_{n}$,

$$
H_{n}\left(v_{0} x\right)= \begin{cases}1, & \text { if } \kappa_{n} Q_{p} \ll 1 \text { or } \chi_{0} \gg 1 \\ \frac{4\left(1+x^{3} \chi_{0}^{3}\right)}{\kappa_{n} Q_{p}}, & \text { if } \kappa_{n} Q_{p} \gg 1 \\ \frac{4}{4+\kappa_{n} Q_{p}}, & \text { if } \chi_{0} \ll 1\end{cases}
$$

one finds

$$
D_{n \ell}= \begin{cases}L_{\ell}, & \text { if } \kappa_{n} Q_{p} \ll 1 \text { or } \chi_{0} \gg 1 \\ \frac{4 L_{\ell-1}}{\kappa_{n} Q_{p},}, & \text { if } \kappa_{n} Q_{p} \gg 1 \\ \frac{4 L_{\ell}}{4+\kappa_{n} Q_{p}}, & \text { if } \chi_{0} \ll 1\end{cases}
$$

Numerical calculations show that $D_{n \ell}$ is a reasonably smooth function of $Q_{p}$ and $\chi_{0}$. The simplest Padé form of $D_{n \ell}$ satisfying Eq. (B4) is thus

$$
D_{n \ell}=\frac{4 L_{\ell-1}}{4 L_{\ell-1}+\kappa_{n} Q_{p} L_{\ell}} L_{\ell} .
$$

This simple approximation is found in excellent agreement with the numerical results. For a wide range of $\kappa_{n} Q_{p}$ and $\chi_{0}$, the errors are within $5 \%$.

One can then obtain

$$
\left\langle\frac{1}{h^{2}}\right\rangle \sum_{n=1}^{\infty} \gamma_{n}\left(1-\frac{1}{\kappa_{n}}\right) U_{2 n} \simeq S \tau_{s} v_{0} \sum_{\ell=1}^{2}\left(\frac{f_{c}^{d}-f_{c}^{p}}{1+\frac{Q_{p} L_{\ell}}{4 f_{c}^{p} L_{\ell-1}}}\right) L_{\ell} X_{\ell}
$$


In order to make the usage of Eq. (B6) straightforward, good Padé approximations of $f_{c}^{d}$ and $f_{c}^{p}$ are also desirable. Using the asymptotic behaviors at $\epsilon \ll 1$,

$$
\left\{\begin{array}{l}
-\frac{3}{4} \int_{0}^{\lambda_{c}} d \lambda\left(\frac{\partial|| \xi|\rangle}{\partial \lambda}\right)^{-1} \simeq 1-1.6 \epsilon^{3 / 2} \\
\frac{3}{4} \int_{0}^{\lambda_{c}} d \lambda \frac{\lambda}{\langle|\xi|\rangle} \simeq 1-1.46 \epsilon^{1 / 2}
\end{array}\right.
$$

and at $\epsilon \rightarrow 1$

$$
\left\{\begin{array}{l}
-\frac{3}{4} \int_{0}^{\lambda_{c}} d \lambda\left(\frac{\partial\langle|\xi|\rangle}{\partial \lambda}\right)^{-1} \simeq 1.5(1-\epsilon) \\
\frac{3}{4} \int_{0}^{\lambda_{c}} d \lambda \frac{\lambda}{\langle|\xi|\rangle} \simeq \frac{3}{8}(1-\epsilon)^{2}
\end{array}\right.
$$

Eqs. (21)-(22) yield the Padé approximations for $f_{c}^{d}$ and $f_{c}^{p}$

$$
\left\{\begin{array}{l}
f_{c}^{d} \simeq \frac{\left(1-\epsilon^{2}\right)^{-1 / 2}\left(1-\epsilon^{2}\right)}{1+1.6 \epsilon^{3 / 2}-1.25 \epsilon^{2}} \\
f_{c}^{p} \simeq \frac{\left(1-\epsilon^{2}\right)^{-1 / 2}(1-\epsilon)^{2}}{1+1.46 \epsilon^{1 / 2}+0.2 \epsilon}
\end{array}\right.
$$

Here, $\left(1-\epsilon^{2}\right)^{-1 / 2}$ comes from $\left\langle h^{-2}\right\rangle$ in a concentric circular equilibrium. The results of $f_{c}^{d}$ and $f_{c}^{p}$, calculated using both the Pade form Eq. (B9) and the exact numerical integration of Eqs. (21)-(22), are given in Fig. (1). It shows the excellent accuracy of this Padé approximation.

Furthermore, by using Eq. (B9), Eq. (B6) is evaluated and compared with the numerical evaluations (using the eigenfunctions $\lambda_{n}$ for $n=1,2,3,4,5$ obtained in Ref. 4). For a wide range of $Q_{p}, \chi_{0}$, and $\epsilon$, the differences are always within $10 \%$, and the analytic approximate results are always larger than the numerical results (which is expected owing to the fact that only eigenfunctions with $n \leq 5$ have been included in the numerical calculations). This shows that the Padé forms given in Eqs. (B6) and (B9) are quite accurate. 


\section{References}

${ }^{1}$ K. Tomabechi, Plas. Phys. Contr., Nucl. Fusion Res. 3, 214, IAEA, Vienna, 1989.

${ }^{2}$ A. Nocentini, M. Tesarotto, F. Engelmann, Nucl. Fusion 15, 359 (1975).

${ }^{3}$ V. Ya. Goroboroko, Ya. I. Kolesnichenko, V. A. Yavorskij, Nucl. Fusion 23, 399 (1983).

${ }^{4}$ C. T. Hsu, P. J. Catto, D. J. Sigmar, Phys. Fluids B2, 280 (1990).

${ }^{5}$ S. P. Hirshman, D. J. Sigmar, Nucl. Fusion 21, 1079 (1881).

${ }^{6}$ C. T. Hsu, D. J. Sigmar, Plasma Phys. Contr. Fus. 32, 499 (1990).

${ }^{7}$ J. W. Connor, J. G. Cordey, Nucl. Fusion 14, 185 (1974).

${ }^{8}$ L. Spitzer, R. Härm, Phys. Rev. 89977 (1953).

${ }^{9}$ R. D. Hazeltine, A. A. Ware, Plasma Phys. 20, 673 (1978).

${ }^{10}$ F. L. Hinton, R. D. Hazeltine, Rev. Mod. Phys. 48, 239 (1976). 


\begin{tabular}{|c|c|c|c|}
\hline & $\begin{array}{c}\text { zeff }=1.5 \\
(f l a t)\end{array}$ & $\begin{array}{c}\text { Zeff }=2.2 \\
\text { (flat) }\end{array}$ & $\begin{array}{c}\text { Zeff }(r) \\
\text { peaked }\end{array}$ \\
\hline$I_{\text {tot }(\mathrm{MA})}$ & 19.03 & 18.64 & 18.90 \\
\hline$I_{(0) \text { (MA) }}$ & 5.34 & 5.05 & 5.23 \\
\hline$I(\alpha)$ (MA) & 0.385 & 0.303 & 0.372 \\
\hline
\end{tabular}

Table 1. Induced bootstrap currents for three different choices of zeff. Input parameters are for the steady state ITER machine. Ohmic seed current is fixed at $13 \mathrm{MA}$. 


\begin{tabular}{|c|c|c|}
\hline & $\begin{array}{c}\text { pitch } \\
\text { angle } \\
\text { scattering } \\
\text { switched off }\end{array}$ & $\begin{array}{c}\text { Fuel } \\
\text { dilution } \\
\text { switched off }\end{array}$ \\
\hline$I_{\text {tot (MA) }}$ & 18.96 & 19.33 \\
\hline$I(0)$ (MA) & 5.36 & 5.54 \\
\hline$I(\alpha)$ (MA) & 0.294 & 0.471 \\
\hline
\end{tabular}

Table 2. Induced bootstrap currents (for zeff $=1.5$ ) produced after the effects of pitch angle scattering and fuel dilution are individually switched off. Input parameters are for the steady state ITER machine. Ohmic seed current is fixed at 13 MA. 


\section{Figure Captions}

Fig. 1: Circulating particle fraction $f_{c}$ in the "pitch angle scattering" dominant and "drag" dominant cases. Padé approximations are in excellent agreement with the exact solution.

Fig. 2: Effective fraction of trapped alphas, assuming four different birth energies $E_{0}$.

Fig. 1: Poloidal magnetic field profile, with and without the effects of alpha bootstrap current. $Z_{\text {eff }}=1.5$.

Fig. 4: Alpha particle induced bootstrap current density as a function of $r / a . Z_{\text {eff }}=1.5$.

Fig. 5: Background bootstrap current density as a function of $r / a . Z_{\text {eff }}=1.5$.

Fig. 6: Fraction of alpha bootstrap current density over background bootstrap current density, for flat $Z_{\text {eff }}=1.5$ and centrally peaked $Z_{\text {eff }}(r)=0.7 \exp \left(-16 r^{2} / a^{2}\right)+$ 1.5 . 


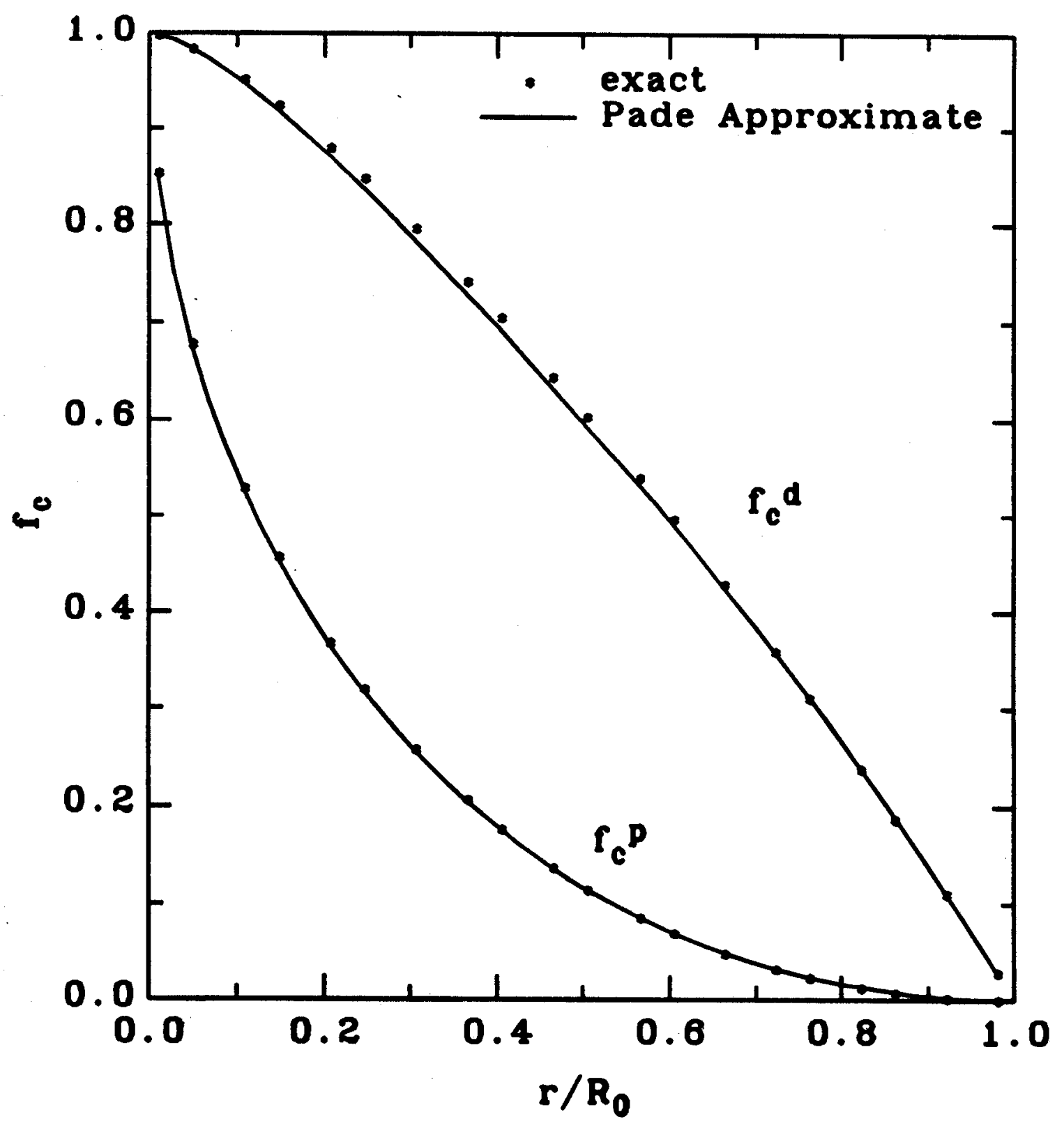

Figure 1 


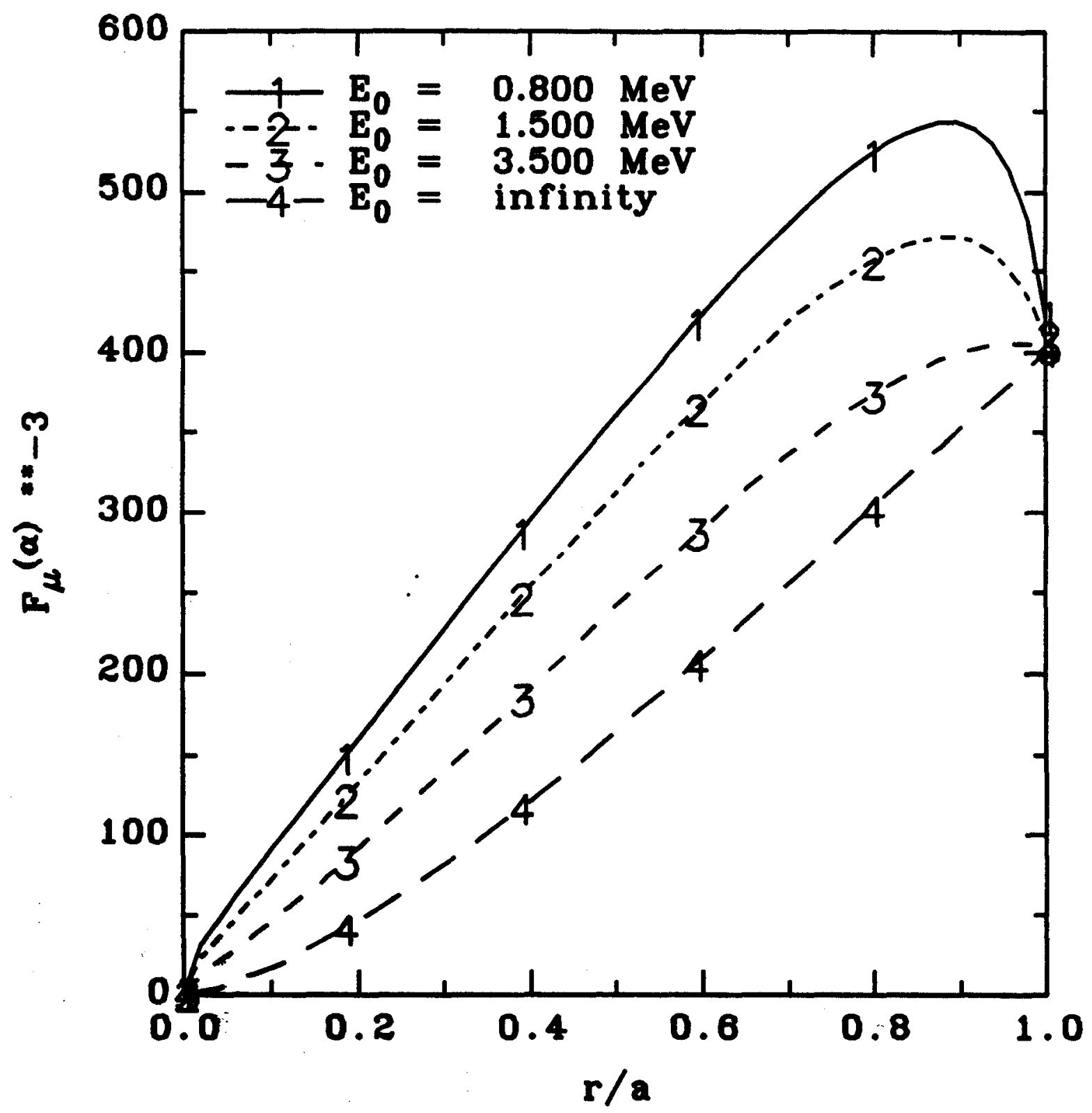

Figure 2 


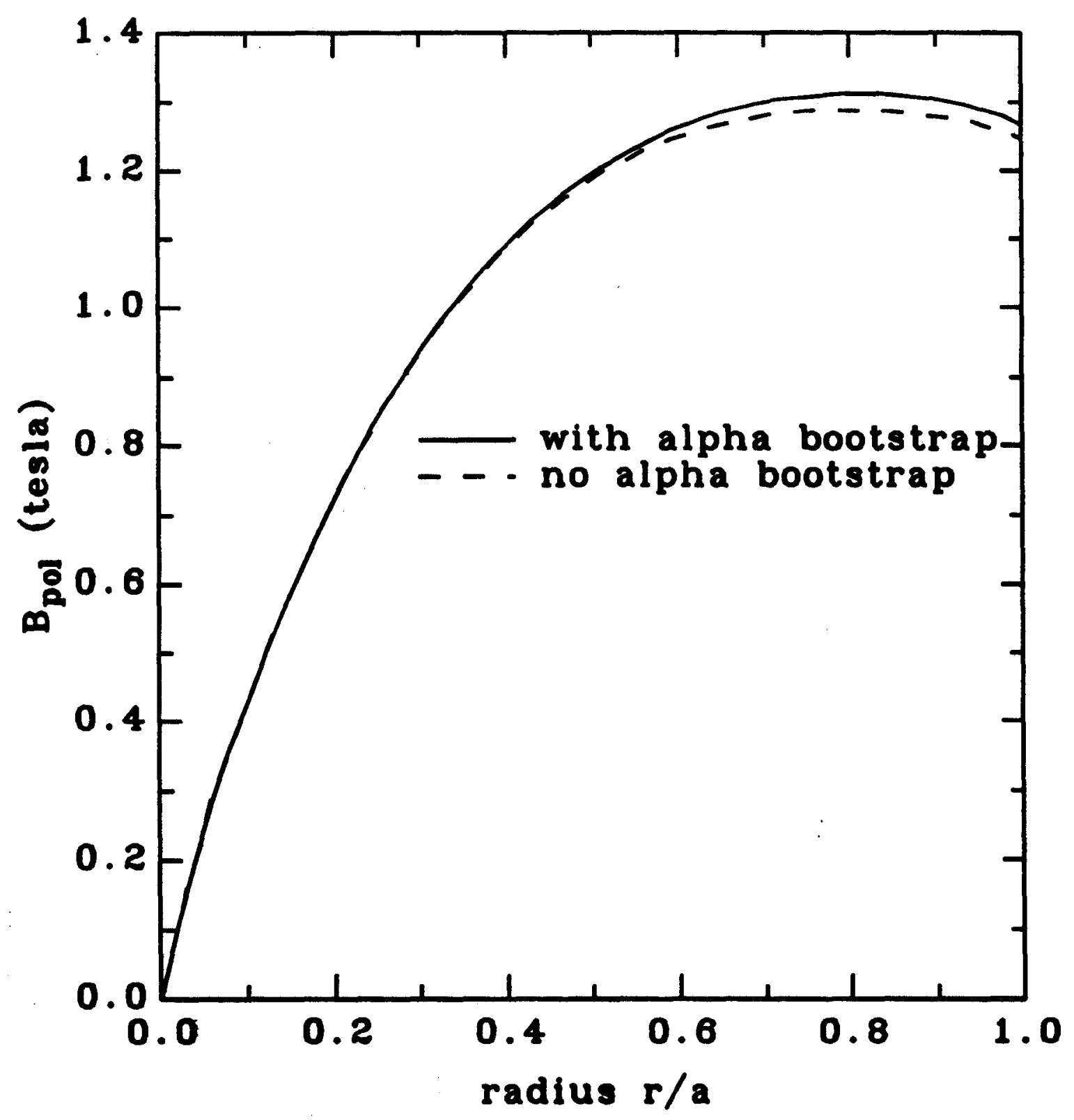

Figure 3 


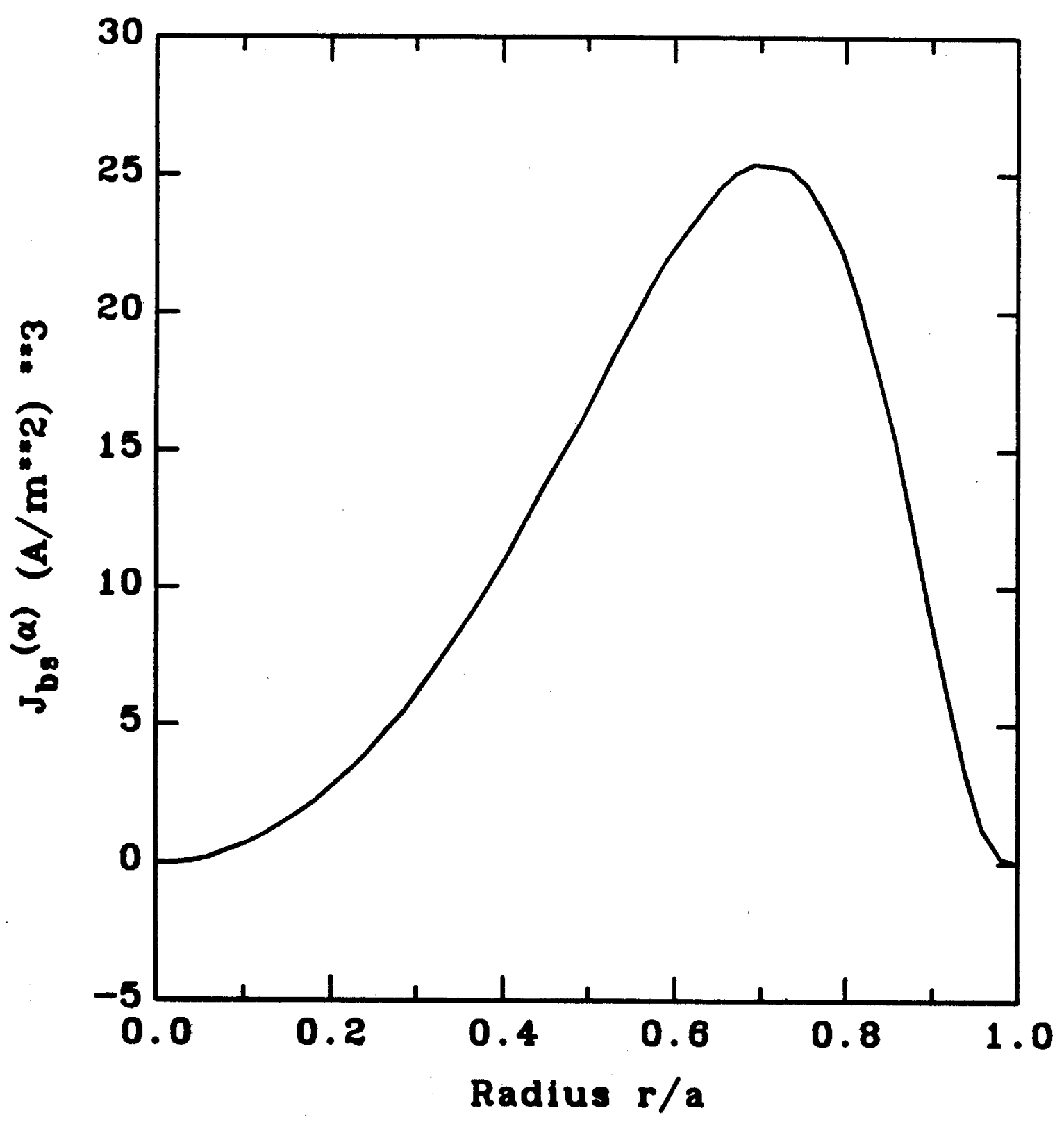

Figure 4 


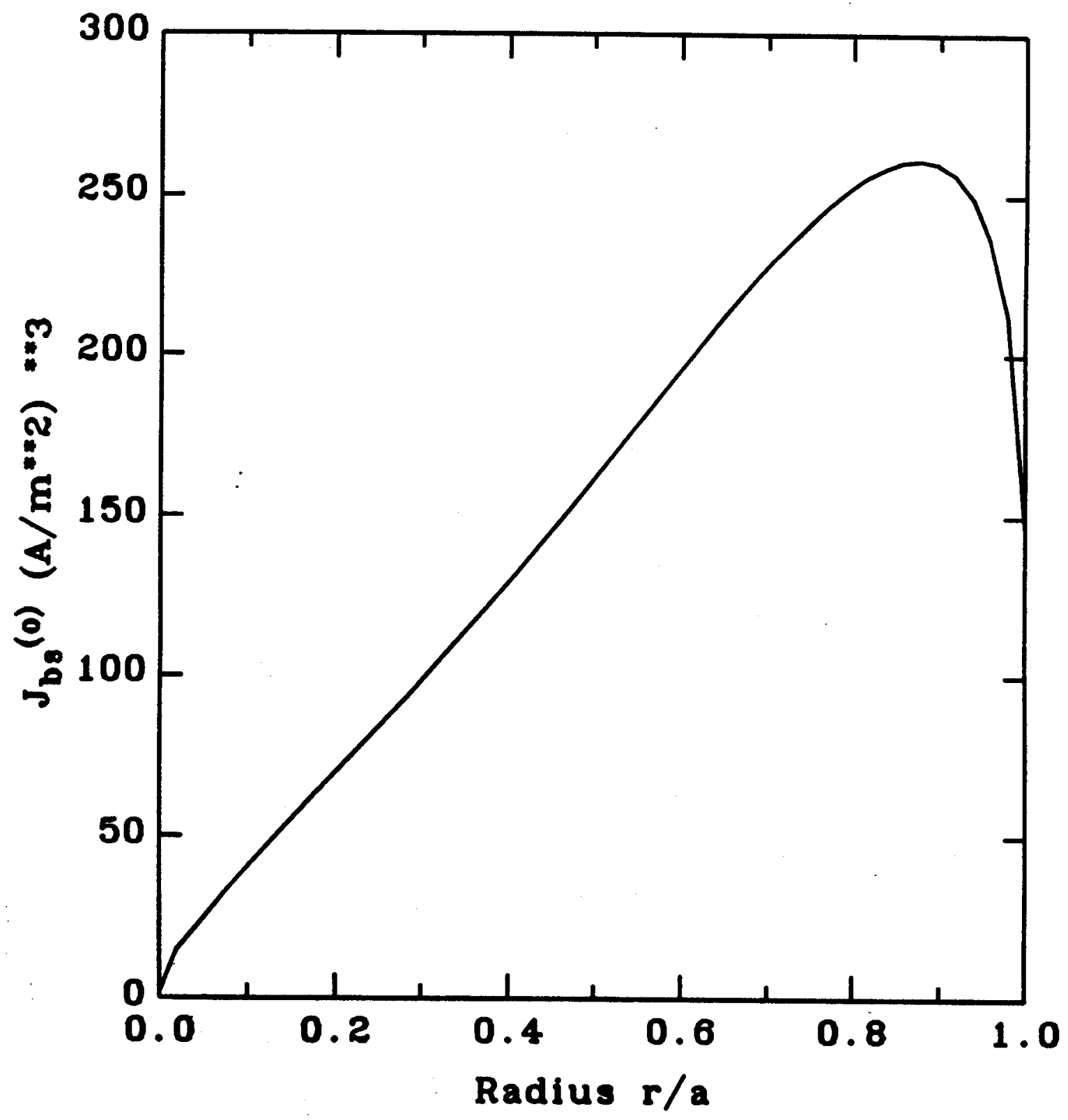

Figure 5 


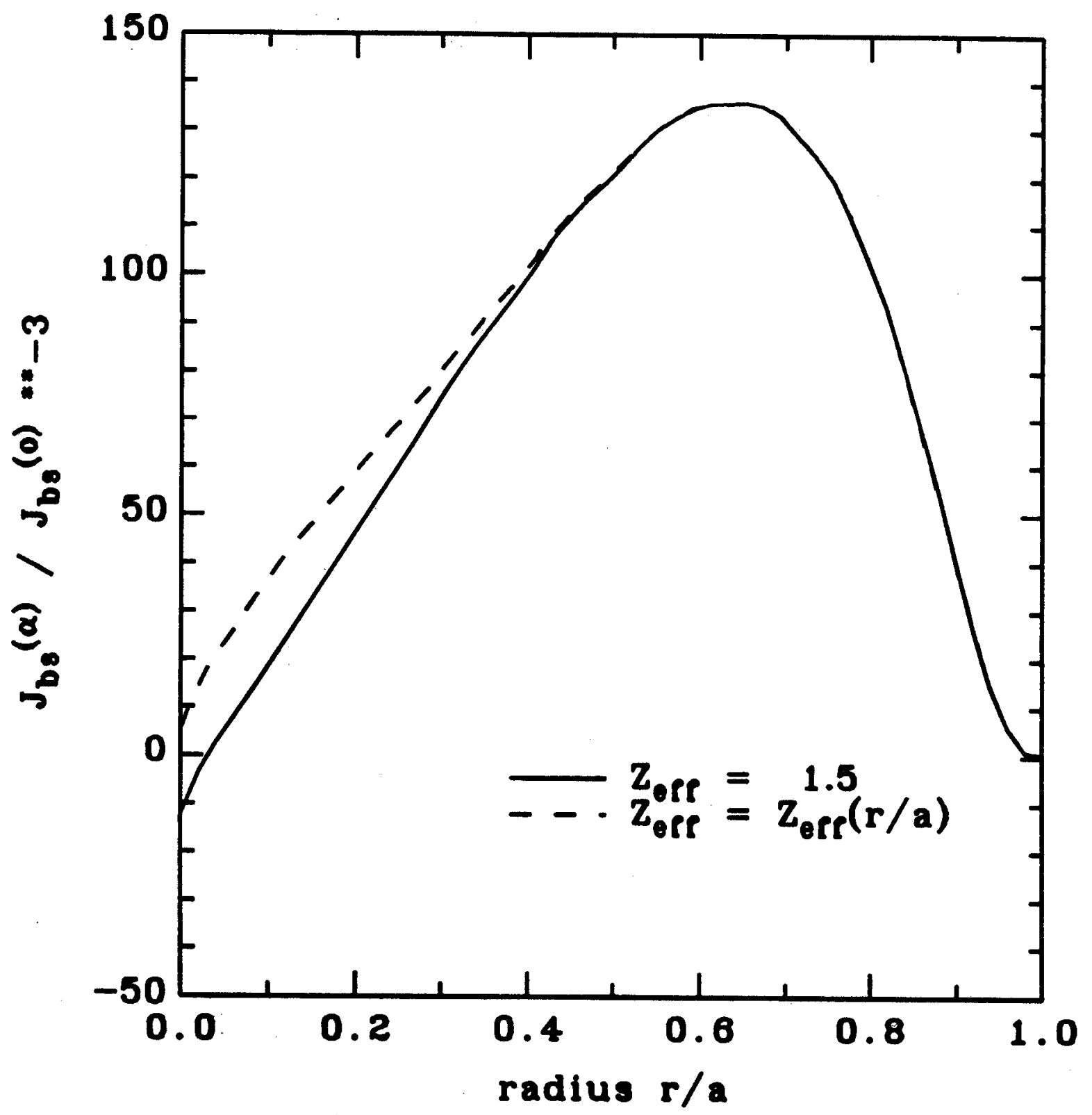

Figure 6 